\title{
A utilização do correio electrónico na comunicação com os utentes da USF Porta do Sol
}

Carla Ponte*

\section{RESUMO}

Objectivo: Caracterizar as mensagens enviadas por correio electrónico entre utentes e médicos e entre utentes e secretários clínicos de uma Unidade de saúde familiar (USF).

Tipo de Estudo: Estudo transversal.

Local: USF Porta do Sol, Matosinhos.

População: Mensagens de correio electrónico trocadas entre os oito médicos e utentes e entre os seis secretários clínicos e utentes da USF.

Métodos: Análise do correio electrónico entre Setembro de 2009 e Fevereiro de 2010. Foram estudadas variáveis relativas ao sujeito que envia a mensagem, o assunto abordado, o tempo entre a mensagem e respectiva resposta e o horário de envio da mensagem.

Resultados: Foram recebidas pelos médicos 594 mensagens (uma média de 74,2 mensagens por médico). Os assuntos mais frequentemente abordados foram as questões médicas, questões administrativas e os meios auxiliares de diagnóstico. A maioria das mensagens abordava apenas um assunto (84,9\%). A maioria das respostas $(50,1 \%)$ foi realizada entre 0 a $12 \mathrm{~h}$ após a recepção da mensagem. Relativamente ao horário de envio das mensagens dos utentes, $85,9 \%$ foram enviadas durante o horário de funcionamento da USF.

Pelo secretariado foram recebidas 49 mensagens. Os assuntos mais frequentemente abordados foram questões administrativas como marcação de consulta (58,3\%). Relativamente ao intervalo de tempo mais frequente entre recepção da mensagem e a resposta foi entre $0-12 \mathrm{~h}(57,1 \%)$. Todas as mensagens foram enviadas durante o horário de funcionamento da USF, assim como as respostas.

Conclusão: Relativamente às mensagens dirigidas aos médicos, o número e tipo de assuntos abordados são coincidentes com estudos já publicados. No que diz respeito às mensagens dirigidas ao secretariado não há dados publicados para comparação; é um estudo pioneiro neste aspecto e ajuda a compreender a importância do correio electrónico como ferramenta de comunicação nos serviços de saúde.

Palavras-chave: Correio Electrónico; Comunicação; Cuidados de Saúde Primários.

\section{INTRODUÇÃO}

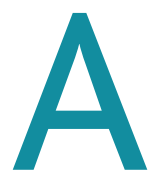
utilização do e-mail para questões de saúde tem sido crescente, sendo objecto de estudo de vários autores, que caracterizaram e avaliaram o conteúdo dos e-mails trocados entre utentes e médicos. ${ }^{2,3,4,6-15}$

*Médica de Família, Unidade de Saúde Familiar Porta do Sol, Unidade Local de Saúde de Matosinhos.
Dada a utilidade desta ferramenta em cuidados de saúde, várias entidades têm elaborado guidelines para a utilização do correio electrónico com os utentes. ${ }^{16,17,18}$

O correio electrónico apresenta vantagens relativamente ao telefone: maior liberdade de acesso, menor custo, a informação não se perde, as mensagens podem ser imprimidas ou guardadas integralmente, as mensagens não têm limite de palavras, permite a discussão do assunto com familiares ou com colegas por parte dos 
utentes e dos profissionais respectivamente, evita interrupções, permite difusão de informação de educação para a saúde para vários utentes, facilita a comunicação na abordagem de questões mais sensíveis. ${ }^{1,2}$

As desvantagens deste meio de comunicação vão sendo identificadas pelos utilizadores: preocupação com a confidencialidade das mensagens, a possibilidade do médico não interpretar correctamente o conteúdo das mesmas, receio do médico não responder. ${ }^{2}$ Relativamente aos profissionais de saúde, estes receiam que esta ferramenta de comunicação vá aumentar o trabalho não contabilizado e não remunerado; no entanto estudos realizados demonstram que a sua utilização não sobrecarrega o profissional, podendo resolver algumas questões de forma mais eficaz que o telefone ou consulta presencial. ${ }^{3}$

Apesar dos receios de sobrecarga de trabalho, os médicos apresentam satisfação considerável relativamente ao uso do correio electrónico. ${ }^{4,5,6}$ Os utentes utilizadores também revelam satisfação alta, sendo esta proporcional à rapidez de resposta. ${ }^{5}$

Em Portugal foram realizados estudos acerca da utilização do correio electrónico na comunicação dos utentes com o médico de família. Estes revelaram uma utilização crescente deste meio de comunicação. Os motivos mais abordados nas mensagens foram questões administrativas e questões médicas. ${ }^{19,20}$

Na USF Porta do Sol foram criadas contas de correio electrónico para todos os médicos e enfermeiros e uma conta global direccionada aos secretários clínicos. O objectivo foi criar maior acessibilidade a todos os profissionais de saúde, tornando estreita a relação do utente com a USF.

A USF é constituída por 8 médicos, 8 enfermeiros e 6 secretários clínicos. Teve início da sua actividade a 30 de Junho de 2009. Em Fevereiro de 2010 tinha inscritos 13681 utentes que pertencem às freguesias de Leça do Balio, S. Mamede Infesta e Custóias.

A criação dos endereços electrónicos foi simultânea, mas a divulgação dos endereços dos médicos teve maior dimensão pela sua inclusão nos horários médicos disponibilizados para consulta dos utentes.

O objectivo deste estudo foi analisar todas as mensagens de correio electrónico enviadas para os profissionais da USF e as respectivas mensagens de resposta, de forma a caracterizar:
- a afluência de mensagens recebidas

- o tipo de assunto abordado

- a rapidez de resposta

- tipo de acção desencadeada pela mensagem.

\section{MÉTODOS}

Foi realizado um estudo transversal sobre as mensagens de correio electrónico trocadas entre os médicos e utentes e entre os secretários clínicos e utentes da USF, entre Setembro de 2009 e Fevereiro de 2010.

Foram recolhidas todas as mensagens recebidas e enviadas pelos 8 médicos de família em actividade na USF, contabilizadas e registadas as variáveis a estudar em Excel. Foi realizada também a contabilização dos dias efectivos de trabalho dos 8 médicos.

Relativamente às mensagens recebidas pelo secretariado, foram contabilizadas e estudadas; foi contabilizado o número de dias efectivos de trabalho dos 6 secretários clínicos.

Quanto às mensagens recebidas pelos enfermeiros não foram incluídas pelo número reduzido de mensagens, devido à divulgação mais tardia dos endereços electrónicos destes profissionais. $\mathrm{O}$ facto de as enfermeiras realizarem maior número de domicílios e disponibilizarem o número de telemóvel de serviço pode contribuir para uma menor necessidade de utilização do correio electrónico para comunicação com os utentes.

Variáveis em estudo: Sujeito que escreve a mensagem e sua relação com o utente sobre o qual se expõe o assunto: próprio, cônjuge, filhos, pais; número e tipo de assuntos abordados, segundo uma adaptação de designações utilizadas em estudos semelhantes: ${ }^{14,15} \mathrm{ac}$ tualizações de referenciações (troca de informação sobre a evolução dos cuidados por outras especialidades); medicação (prescrição inicial, renovação de prescrições crónicas, prescrição de tratamentos de Medicina Física e Reabilitação (MFR) e questões sobre prescrições efectuadas); questões médicas (pedidos de informação e/ou de opinião sobre situações clínicas, pedidos de referenciação); meios complementares de diagnóstico (MCD): fornecimento ou pedido de fornecimento de resultados de MCD, pedido de realização de MCD; questões administrativas ou de enfermagem, marcação e desmarcação de consultas, incluindo domiciliárias; pedidos de atestados / declarações médicas; 
agradecimentos; outros: assuntos não relacionados com a USF; existência de mensagem de resposta do MF; tempo decorrido entre mensagem enviada e respectiva resposta do MF, excluindo fim de semana e férias (entre 0 e 12 horas, mais de doze e menos de 48 horas, 48 horas ou mais); hora de envio das mensagens recebidas e das respectivas respostas (dentro de horário de funcionamento da USF, fora do horário de funcionamento da USF); resultado das mensagens trocadas (ausência de qualquer acção, emissão de receitas, credenciais para MCD ou tratamento ou atestados, informações clínicas e aconselhamento, marcação de consulta, encaminhamento para outro profissional (enfermeiro, secretário clínico), telefonema, outros (actualização de dados identificação).

Durante o período do estudo, ocorreu um problema informático com a perda das mensagens recebidas por um dos médicos durante 1 mês. Foram incluídas as mensagens recebidas no mês seguinte.

\section{RESULTADOS}

\section{Fluxo de mensagens entre utentes e médicos}

Nos 6 meses de estudo foram recebidas 594 mensagens, o que dá uma média de 74,2 mensagens por médico (com um máximo de 142 mensagens e um mínimo de 6 mensagens recebidas). O período do estudo correspondeu a 121 dias de trabalho efectivo, o que perfaz uma média de 4,9 mensagens por dia ( 0,6 mensagens por médico por dia de trabalho).

As mensagens foram enviadas por 249 utilizadores diferentes, o que dá uma média de 2,4 mensagens por utilizador.

O número de mensagens recebidas e o número de utentes utilizadores do e-mail foi diferente entre os médicos; aquele que recebeu menos mensagens $(n=6)$ apresentou 3 utentes utilizadores; o médico que recebeu mais mensagens $(\mathrm{N}=142)$ apresentou 61 utentes utilizadores.

A recepção de mensagens teve uma tendência crescente, com um volume inicial de 28 mensagens no mês de Setembro de 2009 e com 79 mensagens recebidas durante o mês de Fevereiro de 2010.

As mensagens de resposta via e-mail foram no total 469, o que corresponde a 58,6 mensagens por médico. Considerando os 121 dias de trabalho efectivo, equivale a 4,8 mensagens por dia.
Fluxo de mensagens entre os utentes e o secretariado

Foram recebidas 49 mensagens entre 29 de Setembro de 2009 e 28 de Fevereiro de 2010. A maioria das mensagens continha apenas um assunto $(97,9 \%)$. Considerando o tempo efectivo de trabalho desde o período de inicio de recepção de mensagens, obteve-se uma média de 0,49 mensagens por dia.

A recepção de mensagens pelo secretariado tem sido crescente, iniciando com 2 mensagens no mês de Setembro e atingindo 16 mensagens recebidas no mês de Fevereiro.

Foram enviadas 21 mensagens de resposta, realizados 2 telefonemas e encaminhadas as restantes mensagens para os médicos ou enfermeiros para resposta mais direccionada à questão colocada.

\section{Caracterização das mensagens enviadas para os médicos}

Os utilizadores são na maior parte das vezes o próprio (72,8\%), em $4,1 \%$ o cônjuge, $5,5 \%$ o filho e em $17,6 \%$ outro familiar. Em $70 \%$ dos casos, as mensagens foram enviadas por mulheres (quadro I).

Os assuntos mais frequentemente abordados foram as questões médicas, questões administrativas e os meios auxiliares de diagnóstico (quadro I).

A maioria das mensagens abordava apenas um assunto $(84,9 \%), 14,4 \%$ continham dois assuntos e as restantes continham 3 ou 4 assuntos.

$23 \%$ das mensagens não foram respondidas via electrónica pois eram mensagens de agradecimento ou geraram outro tipo de resposta como a resposta telefónica.

As mensagens que obtiveram resposta através de mensagem electrónica foram 469; esse número de mensagens gerou 499 acções, o que corresponde a uma média de 1,06 acções por mensagem recebida. No Quadro I estão representados os tipos de acção desenvolvida, com a respectiva frequência.

A maioria das respostas $(50,1 \%)$ foram realizadas 0 a 12h após a recepção da mensagem, 30,5\% entre 12 e $48 \mathrm{~h}$ e $19,4 \%$ após $48 \mathrm{~h}$. Relativamente ao horário de envio das mensagens dos utentes, $85,9 \%$ foram enviadas durante horário de funcionamento da USF, as restantes fora desse horário. Das mensagens de resposta, $87,2 \%$ foram enviadas no horário de funcionamento da USF. 


\begin{tabular}{|c|c|c|}
\hline \multicolumn{3}{|c|}{$\begin{array}{l}\text { QUADRO I. Caracterização das mensagens recebidas } \\
\text { pelos médicos. }\end{array}$} \\
\hline Sujeito & $\mathbf{n}$ & $\%$ \\
\hline Próprio & 435 & 73,2 \\
\hline Cônjuge & 24 & 4 \\
\hline Filho & 32 & 5,4 \\
\hline Outro Familiar & 103 & 17,4 \\
\hline Sexo & $\mathbf{n}$ & $\%$ \\
\hline Feminino & 416 & 70,0 \\
\hline Masculino & 178 & 30,0 \\
\hline Assuntos abordados & $\mathbf{n}$ & $\%$ \\
\hline Actualização clínica & 41 & 6,2 \\
\hline Actualização acerca referenciações & 38 & 5,7 \\
\hline Medicação & 38 & 5,7 \\
\hline Questões médicas & 181 & 27,3 \\
\hline Meios auxiliares diagnóstico & 123 & 18,5 \\
\hline Questões Administrativas & 128 & 19,3 \\
\hline Declarações & 35 & 5,3 \\
\hline Agradecimentos & 62 & 9,3 \\
\hline Outro & 18 & 2,7 \\
\hline Acções desenvolvidas & $\mathbf{n}$ & $\%$ \\
\hline Emissão de Receitas, MCD, Declarações & 98 & 19,0 \\
\hline Informação clínica, aconselhamento & 166 & 32,2 \\
\hline Marcação de consulta & 107 & 20,8 \\
\hline Encaminhar a outro profissional & 24 & 46,6 \\
\hline Referenciação & 15 & 29,1 \\
\hline Sem acção & 27 & 52,4 \\
\hline Outros & 78 & 15,1 \\
\hline
\end{tabular}

\section{Caracterização das mensagens enviadas para o secretariado}

Os utilizadores são na maior parte das vezes o próprio (83,3\%), em $4,2 \%$ o cônjuge e em $14,5 \%$ outro familiar.

Os assuntos mais frequentemente abordados foram questões administrativas, como marcação e remarcação de consulta (58,3\%), em seguida foram os pedidos de medicação crónica (10,4\%) e questões de enfermagem, como o pedido de agendamento de vacinação $(10,4 \%)$.
Relativamente ao intervalo de tempo entre recepção da mensagem e a emissão da resposta, o mais frequente foi ente $0-12 \mathrm{~h}(57,1 \%)$, seguido do intervalo entre as 12 e $48 \mathrm{~h}(23,8 \%)$ e do intervalo superior a $48 \mathrm{~h}(19,1 \%)$. Todas as mensagens foram enviadas durante o horário de funcionamento da USF, assim como as respostas.

As acções desenvolvidas tendo por base a recepção das mensagens de correio electrónico foram: marcação de consulta $(38,5 \%)$, encaminhar a mensagem para médico $(28,2 \%)$, encaminhar a mensagem para enfermeira $(15,4 \%)$, esclarecimentos sobre funcionamento da USF $(10,2 \%)$ e ou outro $(7,7 \%)$ (quadro II).

\section{DISCUSSÃO}

Foram recebidas 594 mensagens pelos médicos e 49 pelos secretários clínicos. O número de mensagens recebido foi crescente ao longo dos meses. $O$ facto de o estudo ser realizado no início de actividade da USF poderá justificar o número reduzido de mensagens recebidas.

Grande parte das mensagens recebidas pelo secretariado clínico teve como assunto principal as questões administrativas como marcação/remarcação de consultas (58,3\%); este dado realça a utilidade deste tipo de comunicação com o secretariado, com resolução de questões sem necessidade de deslocação do utente à USF.

As mulheres são mais utilizadoras do e-mail, o que é concordante com outros estudos já realizados. ${ }^{2,5,9,19,20}$ Também semelhante a outros estudos foi o número de assuntos abordados por mensagem, maioritariamente um único assunto. ${ }^{2,3,5,6,9,11,15,19,20}$

Relativamente ao tipo de assunto abordado, o mais frequente foi relativo a questões médicas, à semelhança dos estudos encontrados (entre $31 \%$ a $75 \%$ )..$^{3,6,9,15} \mathrm{O}$ segundo e terceiro motivos mais frequentes foram as questões administrativas e informações sobre MAD.

Os estudos portugueses sobre este tema apresentaram como tema de e-mail mais frequente as questões administrativas e informações sobre $\mathrm{MAD}^{21} \mathrm{e}$ as questões médicas. ${ }^{19}$

O facto de haver endereço electrónico para os secretários clínicos pode ter tornado as questões administrativas menos frequentes neste estudo, mas ainda ocupam o 2. ${ }^{\circ}$ lugar, o que pode justificar-se com a utilização inicial menos frequente e familiarização com este tipo de comunicação por parte dos utentes. 


\begin{tabular}{|c|c|c|}
\hline \multicolumn{3}{|c|}{$\begin{array}{l}\text { QUADRO II. Caracterização das mensagens recebidas } \\
\text { pelo Secretariado Clínico. }\end{array}$} \\
\hline Sujeito & $\mathbf{n}$ & $\%$ \\
\hline Próprio & 36 & 83,3 \\
\hline Cônjuge & 1 & 4,2 \\
\hline Outro Familiar & 9 & 14,5 \\
\hline Assuntos abordados & $\mathbf{n}$ & $\%$ \\
\hline Marcação de consulta & 23 & 46,9 \\
\hline Remarcações de consultas & 5 & 10,2 \\
\hline Medicação & 5 & 10,2 \\
\hline Esclarecimentos Funcionamento & 4 & 8,2 \\
\hline Assuntos Médicos & 2 & 4,1 \\
\hline Assuntos Enfermagem & 5 & 10,2 \\
\hline Agradecimentos & 4 & 8,2 \\
\hline Outro & 1 & 2 \\
\hline Acções desenvolvidas & $\mathbf{n}$ & $\%$ \\
\hline Marcação consulta & 15 & 38,5 \\
\hline Esclarecimento/Informação & 4 & 10,2 \\
\hline Encaminhar para médico & 11 & 28,2 \\
\hline Encaminhar enfermeiro & 6 & 15,4 \\
\hline Outros & 3 & 7,7 \\
\hline
\end{tabular}

As mensagens recebidas levaram a várias acções médicas, sendo as mais frequentes encaminhar a outro profissional da equipa de saúde $(46,6 \%)$ e esclarecimentos/informação clínica (32,2\%). Comparando com Anand et al, ${ }^{9}$ neste último a maioria das acções foram orientação terapêutica (63\%). No estudo de Mónica Granja, ${ }^{19}$ os actos médicos mais frequentemente realizados em resposta às mensagens electrónicas foram relacionadas com aconselhamento/informação médica $(38,9 \%)$ e emissão de receitas (20\%). Observamos assim semelhança entre estes resultados.

No caso deste estudo obteve-se um elevado número de actos relacionados com o «encaminhar para outro profissional da equipa de saúde», o que pode demonstrar a vontade dos utentes comunicarem com outros membros da equipa de saúde além do médico. Essa necessidade espelha-se na frequência de recepção de mensagens que foram reencaminhadas para esses profissionais e pelos resultados de outros estudos que mos- tram que as questões administrativas são um dos assuntos mais abordados nas mensagens. Na USF já se criaram também endereços electrónicos para enfermeiros, embora tenham sido divulgados mais tarde e não tenham ainda fluxo considerável. Há ainda a referir a importância do endereço electrónico para a área administrativa, pois aumenta a acessibilidade e permite ao utente resolver vários assuntos com maior comodidade. A utilização crescente do endereço electrónico geral da USF (direccionado para os secretários clínicos) pode demonstrar a abertura dos utentes para este tipo de comunicação, com as vantagens que lhe são inerentes.

$\mathrm{Na}$ maior parte das vezes $(50,1 \%)$, o tempo de resposta foi igual ou inferior a $12 \mathrm{~h}$ após a recepção da mensagem. No estudo de Leong et al, ${ }^{6} 38 \%$ das mensagens foram respondidas no próprio dia $(<24 \mathrm{~h})$.

Relativamente às mensagens recebidas pelo secretariado, o número foi mais reduzido, mas tem vindo a ser crescente, resolvendo algumas questões administrativas que eram direccionadas para o médico na primeira fase de funcionamento da USF. Este meio de comunicação que os utentes têm à disposição para resolver questões administrativas é uma mais-valia, uma vez que descongestiona o telefone para chamadas de resolução mais imediata. Permite ainda o envio de mensagem do local de trabalho ou de casa, no horário que mais convém ao utente.

Da bibliografia disponível, não foi encontrado nenhum estudo com a abordagem das mensagens entre utentes e secretários clínicos. Este estudo traz dados novos nesse campo, mostrando que a disponibilidade desse meio de comunicação aumenta a acessibilidade do utente aos serviços de saúde. Como USF, e adoptando um modelo organizacional em equipa multidisciplinar, é essencial uniformizar a acessibilidade aos vários grupos profissionais. Por essa razão foram estudadas as mensagens enviadas a esses grupos (não incluindo nesta primeira fase de funcionamento o grupo de enfermagem pelos motivos já referidos).

Como limitação deste estudo pode-se referir o facto de o estudo decorrer no início de actividade da USF, o que pode ser um motivo de pouca utilização do correio electrónico devido à pouca divulgação inicial do endereço electrónico.

Como ponto forte pode-se apontar o facto de ter sido o mesmo médico a recolher e classificar os motivos das 
mensagens, o que leva a menor viés de classificação. Trata-se de um tema original, com poucos estudos publicados em Portugal, enriquecendo o conhecimento sobre a prática médica neste domínio e o primeiro a estudar as mensagens para os secretários clínicos. Pode servir para alterar a prática médica, com maior interesse por este tipo de comunicação, demonstrando que este começa a ser utilizado amplamente pelos médicos de família e outros profissionais de saúde.

Teria interesse, em estudos futuros, estudar os utilizadores e qual o grau de satisfação dos mesmos. A realização de um estudo mais amplo, englobando um período de tempo maior e numa altura de funcionamento da USF mais estável e madura, poderia dar origem a resultados interessantes e mais representativos da actual actividade da unidade de saúde.

Outro ponto a analisar seria o tempo consumido por esta actividade e qual a opinião dos médicos de família que utilizam este tipo de comunicação com os utentes, no que diz respeito a satisfação e sobrecarga de trabalho adicional. Outra vertente interessante seria desenhar um estudo semelhante incluindo também as mensagens recebidas pelos enfermeiros.

\section{AGRADECIMENTOS}

A autora agradece às colegas que colaboraram na colheita de dados, com a disponibilização das suas mensagens de correio electrónico:

Dra. Ana João Reis, Dra. Andreia Cerejo, Dra. Carla Nina, Dra. Filipa Lobo, Dra. Ilda Gonçalves, Dra. Mariana Tudela e Dr. Sílvia Henriques.

Agradece ao secretário clínico Cláudio Ferreira pela recolha das mensagens do secretariado clínico da USF.

Um agradecimento pela colaboração imprescindível na análise crítica dos resultados à Dra. Mónica Granja.

\section{CONFLITO DE INTERESSES}

A autora é editora da Revista Portuguesa de Clínica Geral desde Março de 2011 e declara não ter estado envolvida no processo de decisão editorial relativo a este artigo.

\section{REFERÊNCIAS BIBLIOGRÁFICAS}

1. Car J, Sheikh A. Email consultations in health care: 2 - acceptability and safe application. BMJ 2004 Aug 21; 329 (7463): 439-42.

2. Houston TK, Sands DZ, Jenckes MW, Ford DE. Experiences of patients who were early adopters of electronic communication with their physician: satisfaction, benefits, and concerns. Am J Manag Care 2004 Sep; 10 (9): 601-8.

3. Sittig DF. Results of a content analysis of electronic messages (e-mail) sent between patients and their physicians. BMC Med Inform Decis Mak 2003 Oct 1; 3: 11.

4. Gaster B, Knight CL, DeWitt DE, Sheffield JV, Assefi NP, Buchwald D.
Physician's use and attitudes toward electronic mail for patient communication. J Gen Intern Med 2003 May; 18 (5): 385-9.

5. Liederman EM, Lee JC, Baquero VH, Seites PG. Patient-physician web messaging: the impact on message volume and satisfaction. J Gen Intern Med 2005 Jan; 20 (1): 52-7.

6. Leong SL, Gingrich D, Lewis PR, Mauger DT, George JH. Enhancing doctor-patient communication using email: a pilot study. J Am Board Fam Pract 2005 May-Jun; 18 (3): 180-8.

7. Neville R, Marsden W, McCowan C, Pagliari C, Mullen H, Fannin A. Email consultations in general practice. Br J Gen Pract 2004 Jul; 54 (504): 546.

8. Hereford J, Bell E, Lee J, Eytan T. Patient satisfaction with a web-based patient-provider messaging system implemented across a statewide delivery system (Group Health Cooperative). AMIA Annu Symp Proc 2003: 860.

9. Anand SG, Feldman MJ, Geller DS, Bisbee A, Bauchner H. A content analysis of e-mail communication between primary care providers and parents. Pediatrics 2005 May; 115 (5): 1283-8.

10. Patt MR, Houston TK, Jenckes MW, Sands DZ, Ford DE. Doctors who are using e-mail with their patients: a qualitative exploration. J Med Internet Res 2003 Apr-Jun; 5 (2): e9. doi: 10.2196/jmir.5.2.e9

11. Liederman EM, Morefield CS. Web messaging: a new tool for patientphysician communication. J Am Med Inform Assoc 2003 May-Jun; 10 (3): 260-70

12. Delbanco T, Sands DZ. Electrons in flight: e-mail between doctors and patients. N Engl J Med 2004 Apr 22; 350 (17): 1705-7.

13. Stone JH. Communication between physicians and patients in the era of E-medicine. N Engl J Med 2007 Jun 14; 356 (24): 2451-4.

14. Gerstle RS; American Academy of Pediatrics Task Force on Medical Informatics. E-mail communication between pediatricians and their patients. Pediatrics 2004 Jul; 114 (1): 317-21.

15. White CB, Moyer CA, Stern DT, Katz SJ. A content analysis of e-mail communication between patients and their providers: patients get the message. J Am Med Inform Assoc 2004 Jul-Aug; 11 (4): 260-7.

16. Kane B, Sands DZ. Guidelines for the clinical use of electronic mail with patients. J Am Med Inform Assoc 1998 Jan-feb; 5 (1): 104-11.

17. Physician Guidelines for Online Communication with Patients. Ottawa: Canadian Medical Association; 2005.

18. American Medical Association. Guidelines for Physician-Patient Electronic Communications. Disponível em: http://www.amaassn.org/ama/pub/category/2386.html [acedido em 09/06/2008].

19. Granja M. O uso do e-mail na comunicação com o médico de família: catorze meses de experiência. Rev Port Clin Geral 2009 Nov-Dez; 25 (6): 639-46.

20. Pinhão R, Calisto M, Pimentel MA, Fernandes R. Implementação da comunicação médico-doente via endereço electrónico na consulta de MGF. Rev Port Clin Geral 2009 Nov-Dez; 25 (6) :634-8.

\section{ENDEREÇO PARA CORRESPONDÊNCIA}

Carla Ponte

USF Porta do Sol

Praceta Recarei de Cima, 56

4465-339 Leça do Balio

carla.ponte.pds@gmail.com

Recebido em 16/09/2010

Aceite para publicação em 15/05/2011 


\section{ABSTRACT}

\section{USE OF ELECTRONIC MAIL IN COMMUNICATION WITH PATIENTS IN USF PORTA DO SOL}

Aim: To characterize the e-mail messages sent between patients and physicians and between patients and medical secretaries in a family health unit (FHU.)

Type of study: Cross sectional study.

Setting: USF Porta do Sol, Matosinhos, Portugal

Population: Electronic mail messages exchanged between the eight doctors and their patients and between the six clinical secretaries and the patients of the family health unit.

Method: An analysis of electronic mail sent between September 2009 and February 2010. The variables studied were the sender of the message, the subject matter of the message, the time between the message and the response and time of sending the message.

Results: We received 549 medical electronic messages (an average of 74.2 per physician) in the study period. The issues most frequently discussed were medical issues, administrative issues and diagnostic tests. Most messages addressed only one subject $(84.9 \%)$. Most responses (50.1\%) were sent between 0 to 12 hours after receipt of the message. $85.9 \%$ of the messages were sent during operating hours of the USF.

The secretaries received 49 messages. The issues most frequently discussed were administrative issues such as appointments (58.3\%). The most frequent time interval between receipt of the message and the response was between $0-12 \mathrm{~h}(57.1 \%)$. All messages and responses were sent during the operating hours of the USF.

Conclusion: For e-mail messages sent to physicians, the number and type of issues discussed are similar to those found in previously published studies. This study presents new data with respect to messages sent to and from medical secretaries. It helps to understand the importance of email as a communication tool in health services.

Keywords: Electronic Mail; Communication; Primary Health Care. 